\title{
Development of LED Road Traffic Signal Considering Washout Phenomenon
}

\author{
${\text { Kazutaka } \mathrm{HONGO}^{\mathrm{a}} * \text {, Kentarou HAYASHI }}^{\mathrm{b}}$, Yoshinori AKINAGA ${ }^{\mathrm{b}}$, Masahiro KOUROGI ${ }^{\mathrm{b}}$ \\ Makoto MIYAUCHI ${ }^{\mathrm{a}}$, and Seiichi SERIKAWA ${ }^{\mathrm{c}}$ \\ ${ }^{a}$ Kitakyushu College of Technology, 5-20-1 Shii, Kokuraminami-ku, Kitakyushu 802-0985, Japan \\ ${ }^{\mathrm{b}}$ Shingo Denzai Co., Ltd., 1-29 Shinkomachi, Omuta, Fukuoka, 836-0061 Japan \\ ${ }^{\mathrm{c}}$ Kyushu Institute of Technology, 1-1 Sensui-cho, Tobata-ku, Kitakyushu, 804-8550, Japan \\ *Corresponding Author: kazu@kct.ac.jp
}

\begin{abstract}
Blue LED is recently mass-produced; road traffic signal head using LED system began to be used. The LED signal head has advantages such as energy saving and long life. However, the newly proposed instrument LED signal head is difficult to reduce problems such as washout phenomenon. In this paper, we have proposed reduction of washout phenomenon of LED signal head by using the LED element 192 and low-reflection black plate on a blank area between the elements. In the measurement of light intensity distribution and luminance uniformity, it was confirmed that the LED type signal head we proposed satisfy the conditions described in the traffic signal lighting specification by the National Police Agency. The visibility of signal head are improved ,as apparent from the picture of before and after improvement when in the morning and evening sun altitude low and result of phantom ratio.
\end{abstract}

Keywords: Road traffic signal head, washout phenomenon., Phantom ratio

\section{Introduction}

The world's first high-brightness blue LED was produced in 1989. Practical technique for manufacturing it has been established in 1993. In this way, the application to the traffic signal has become possible by RGB LED are aligned. In Japan, the experimental operation of road traffic signal LED was started in Aichi Prefecture and Tokushima Prefecture in 1994. In North America, the evaluation of some LED for using to traffic signal, heat and on cost and efficiency issues have been reported, and also the solution to problems that lose of the difference between yellow and red LED traffic signal for color-deficient drivers ${ }^{(1-3)}$. In addition to these problems, the evaluation method for determining the turn on and off in the case with regard to lighting pseudo caused when the sun is irradiated on the surface of a light, the light of high intensity is irradiated to a traffic light during daytime such as has been shown ${ }^{(4)}$. In this paper, we have proposed a road traffic signal lights that are designed to reduce the wash-out phenomenon occurring in the LED road traffic signal system.

\section{LED Road traffic signal head}

\subsection{Washout Phenomenon}

A road traffic signal head using a filament bulb is to turn on the signal light unit, by reflected the light of the source by the reflector and through the colored filter lens. Therefore, in the morning and evening sun altitude low, pseudo-lighting phenomenon looks to be lighting as if it were had occurred by sunlight is irradiated to the reflector through the colored filters ${ }^{(5)}$. On the other hand, in a road traffic signal head using LED, unlike bulb type, pseudo-lighting phenomenon does not occur since no reflection mirror is used. However, as shown in Figure 1, in the morning and evening low solar altitude, and in the type having an uneven small front lens or using light diffusing lens, the color of signal cannot be identified by driver and pedestrian. This phenomenon is called a washout phenomenon. Figure 1 shows the pictures of washout phenomenon.

The guideline represents the ease of visibility of the signal color is phantom ratio. Phantom ratio is the ratio of 
the brightness of the off state and brightness of the lighting state of the signal light unit. Phantom ratio increases, the contrast of the unlit and lit to clear, so the signal color is easily visible.

\subsection{3 Signal head using LED}

Blue LED is now mass-produced, and now available for a lot of road traffic signal head. The road traffic signal head using LED have a high visibility, since a light source of LED elements emit light directly, resulting in uniform light compared to the filament bulb and using no reflector. In addition, in morning and evening sun of low-altitude, unlike bulb type, pseudo-lighting phenomenon does not occur since no reflection mirror is used. Furthermore, the power consumption of LED system is 9.5 [VA] against that of bulb system using TS-70R (70W) traffic signal light bulb. The life of the LED system is considered to be approximately six to eight years, though bulbs have been replaced for about a year, and It is also excellent from the viewpoint of cost savings and inhibiting the flow of traffic due to bulb replacement. However, as shown in Fig. 1, in the morning and evening low solar altitude, and in the type having an uneven small front lens, the color of signal can not be identified due to occurring washout phenomenon. As

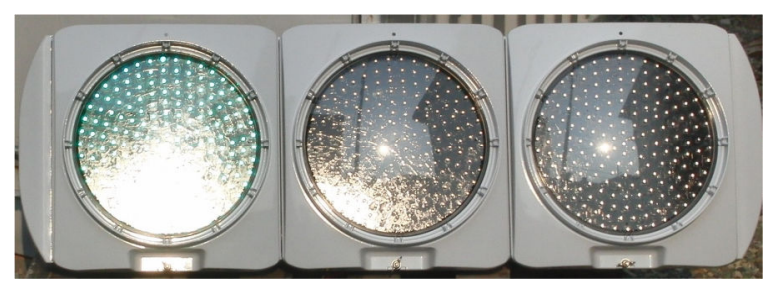

(a) Green lit

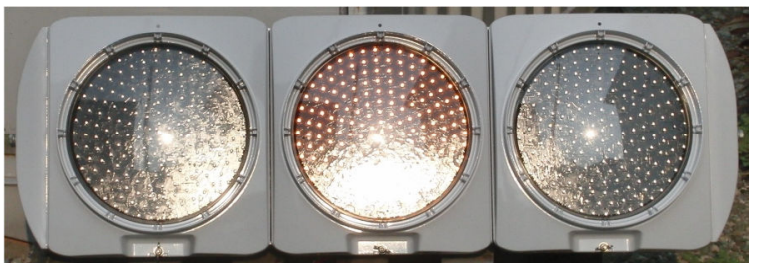

(b) Yellow lit

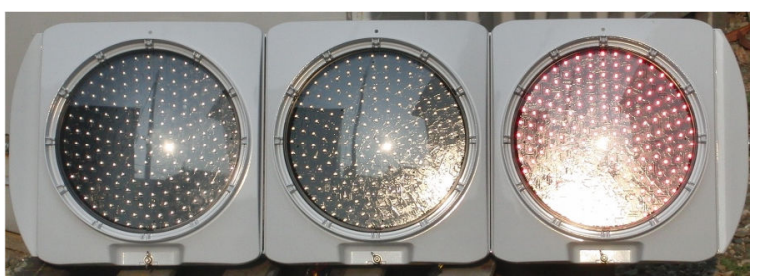

(b) Red lit

Fig. 1. Washout phenomenon a solution, we have proposed reduction of washout phenomenon of LED signal head by using the LED element 192 and low-reflection black board on a blank area between the LED elements. Figure 2 shows the structure of the LED type signal head of reducing-"washout phenomenon" we proposed. The low reflectance plate we called "phantom board" is inserted between the front lens and the LED substrate. Diffused reflection of incident sunlight that

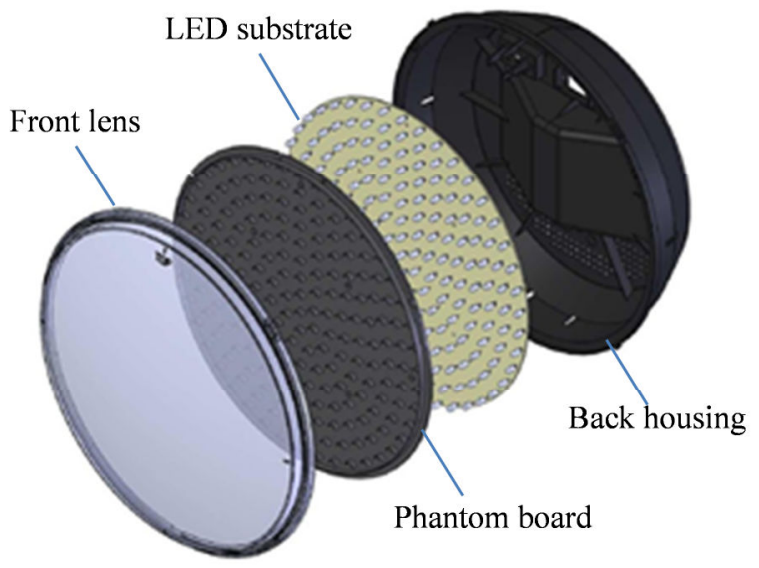

Fig. 2. Structure of the LED traffic signal head for reducing- "washout phenomenon"

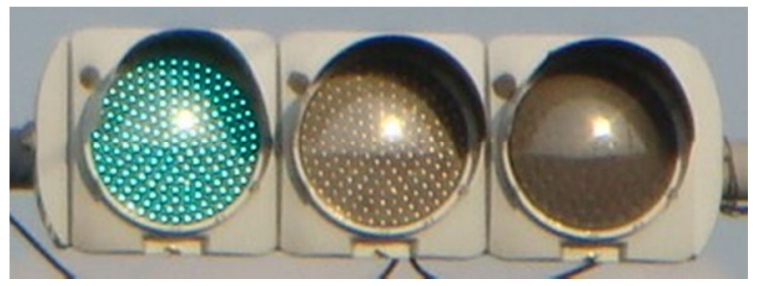

(a) Green lit

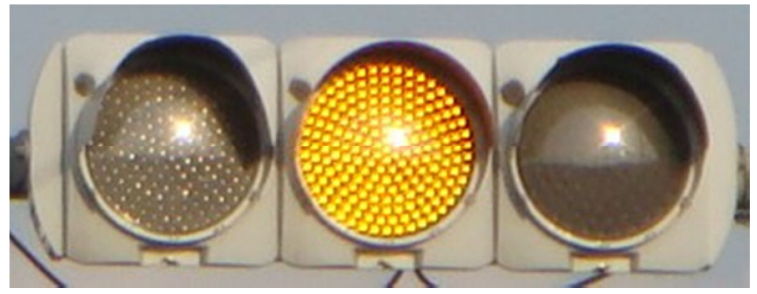

(b) Yellow lit

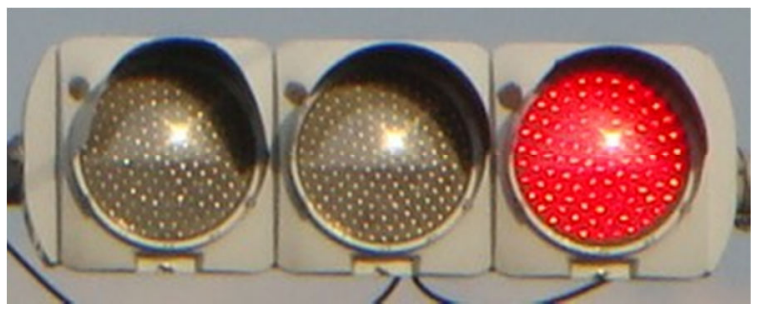

(b) Red lit

Fig 3. LED traffic signal head for reducing"washout phenomenon" 
occurs at front lens and the blank area between LED elements can be suppressed by the phantom board. Therefore, the washout phenomenon is reduced, and the color of the traffic signal lights can be recognized clearly. Figure 3 shows LED traffic signal head we proposed, as apparent from this figure, we can recognize that only green or yellow or red light is lit and other colors are not lit.

\subsection{Measurement and results}

The measurement apparatus of phantom ratio is shown in Figure 4. In this figure, the illuminance of the pseudo sunlight incident on the optical unit is 1000 [lx]. The brightness of signal light when the optical unit is lit and pseudo sun light is not lit, and the brightness of pseudo sunlight reflection when the optical unit is not lit and pseudo sunlight is lit, are measured. The measurement results of phantom ratio are shown in Table 1 and Table 2. From these Tables, however, phantom ratio of non-reduction products is greater than that of measures product. The test conditions of the phantom ratio measures

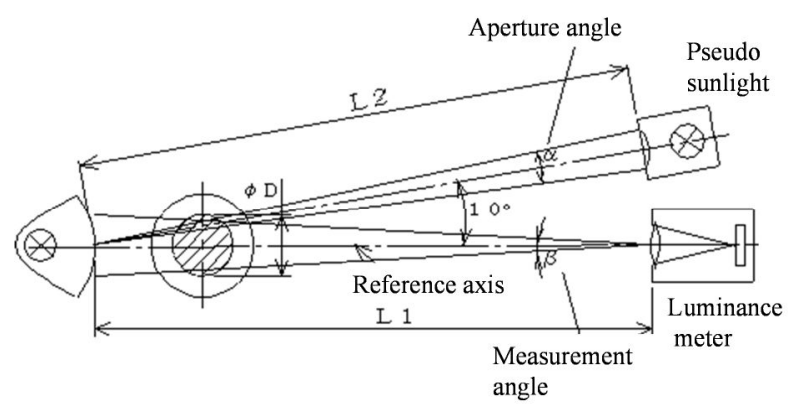

Fig. 4. Measurement of phantom ratio

Table 1. Phantom ratio before improvement

\begin{tabular}{|c|c|c|c|}
\hline & $\begin{array}{c}\text { Lighting } \\
\text { brightness } \\
\left(\mathrm{cd} / \mathrm{m}^{2}\right)\end{array}$ & $\begin{array}{c}\text { Reflection } \\
\text { brightness } \\
\left(\mathrm{cd} / \mathrm{m}^{2}\right)\end{array}$ & Phantom ratio \\
\hline $\mathrm{G}$ & 6551 & 352 & 18.61 \\
\hline $\mathrm{Y}$ & 12859 & 546 & 23.55 \\
\hline $\mathrm{R}$ & 4288 & 765 & 5.61 \\
\hline
\end{tabular}

Table 2. Phantom ratio after improvement

\begin{tabular}{|c|c|c|c|}
\hline & $\begin{array}{c}\text { Lighting } \\
\text { brightness }\end{array}$ & $\begin{array}{c}\text { Reflection } \\
\text { brightness }\end{array}$ & Phantom ratio \\
\hline $\mathrm{G}$ & 6551 & 501 & 13.08 \\
\hline $\mathrm{Y}$ & 12859 & 655 & 19.63 \\
\hline $\mathrm{R}$ & 4288 & 855 & 5.02 \\
\hline
\end{tabular}

the reflected light at a middle position of the exit angle due to reflection from the substrate in an upright angle of incidence of sunlight, so conditions of a regular reflection position by the sunlight, does not take into account. The actual site, it will be painful to visible by light that was reflected by the substrate normal to the incident angle of sunlight, there is provided a phantom board in order to prevent the impact. The effect of the insertion of the phantom board is clear from Figure 3, the visibility is improved. A characteristic of the light intensity distribution was measured by using a digital illuminance meter TOPCON IM-5, according to the traffic signal lighting specification by the National Police Agency. The luminosity standard prescribed by the National Police Agency is shown in Fig. 5. The measurement conditions is as follows; $0^{\circ}$ is the optical axis direction; Input voltage AC 100 [V]; at room temperature $25{ }^{\circ} \mathrm{C}$; Humidity $65 \mathrm{Rh}$. The

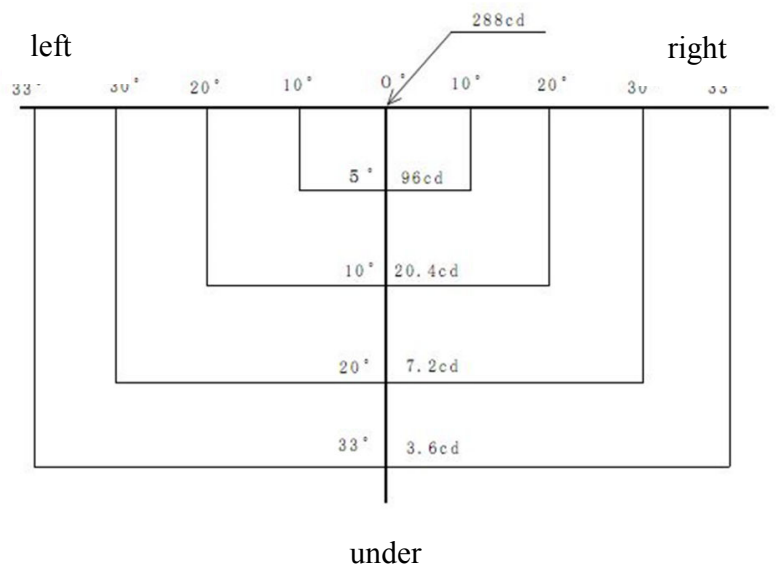

Fig. 5. Standard luminosity

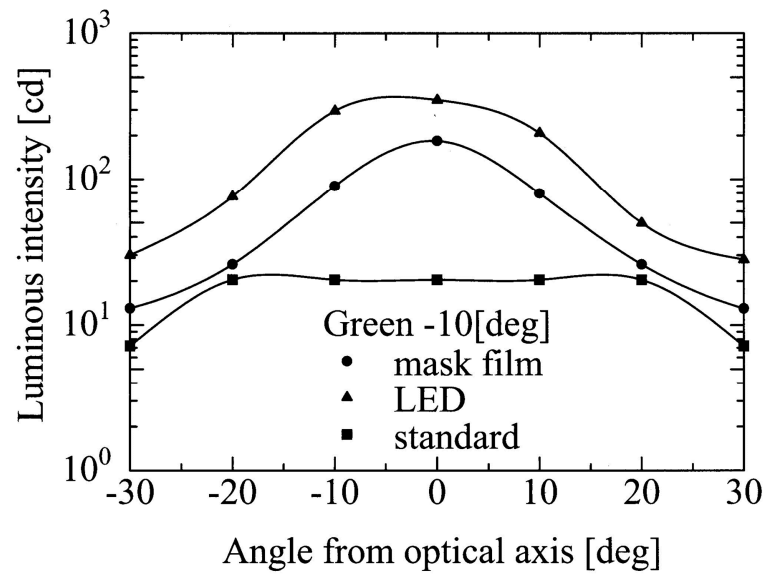

Fig. 6. Characteristics of the light intensity distribution (Green 10[deg]) 
measurement results are shown in Fig. 6. This Figure shows the characteristics of the light intensity distribution in the case of green light at $10[\mathrm{deg}]$. Both of the bulb type and the LED type satisfy the conditions described in the traffic signal lighting specification by the National Police Agency, however the LED method is found to be higher than the light intensity of bulb type.

The measurement of luminance uniformity was carried out by using a luminance colorimeter TOPCON BM-7, according to the procedure described in the traffic signal lighting specification by the National Police Agency. The measurement conditions are the same as those described above. A luminance ratio from the measurements is $1.97: 1$, and are satisfied the condition described in the traffic signal lighting specification by the National Police Agency.

\section{Conclusions}

We have succeeded in reducing the washout phenomenon of LED type signal head by using "phantom board", which is low-reflection black board to prevent diffused reflection of incident sunlight at small lens. The effect of the insertion of the phantom board is clear from pictures when the afternoon sun is striking, the visibility is improved. In the measurement of light intensity distribution and luminance uniformity, it was confirmed that the LED type satisfy the conditions described in the traffic signal lighting specification by the National Police Agency.

As a result, even in the early morning or evening when the sun altitude is low and the strong sunlight is striking, the driver and pedestrians are easily to recognize the color of signal lights

\section{References}

(1) I. Lewin, J. Corbin, M. Janoff : "The Application of Light Emitting Diodes to Traffic Signals", Jouranal of the Illuminating Engineering Society, pp. 17-26(1997)

(2) Conway, K. M. , Bullough, J. D.:"WILL LEDS TRANSFORM TRAFFIC SIGNALS AS THEY DID SIGNS?", Proc. IESNA Annual Conference, pp. 1-10(1999)

(3) Taro Ochiai: "Universal-Design LED Traffic Signals for Color-deficient Drivers", CIE 26th Proceedings, Vol. 2, D4-68-71(2007)

(4) M. Miyauchi, T. Shimomura, M.Kourogi, N.Shinto :
"A Study of Recognition of Pedestrian Traffic Signal Head”, Proc.1999 Annual Conf. IEI Jpn., 32pp. 239

(5) M. Miyauchi, T. Shimomura, M.Kourogi, N.Shinto : " Road Traffic Signal Head with Mask-Film-Type Lens-Unit”, J. Illum. Engng. Jpn., 84(5), pp. 296-301 